\title{
VANISHING MAGNETIC FIELD LIMITS OF SOLUTIONS TO THE PRESSURELESS MAGNETOGASDYNAMICS
}

\author{
HongJun CHENG AND ZHOUHONG Sun
}

\begin{abstract}
We are interested in the system of conservation laws modeling the pressureless magnetogasdynamics. Firstly, we solve the Riemann problem and obtain five kinds of structures consisting of combinations of shocks, rarefaction waves and contact discontinuities. Secondly, we study the vanishing magnetic field limits of the Riemann solutions to the pressureless magnetogasdynamics and show that the density and velocity in the Riemann solutions to the pressureless magnetogasdynamics converge to the Riemann solutions to the pressureless gas dynamics. The formation processes of delta-shocks and vacuum states are discussed in details.
\end{abstract}

Mathematics subject classification (2010): 35L65, 35B30, 35L67, 35B25.

Keywords and phrases: Pressureless magnetogasdynamics, pressureless gas dynamics, vanishing magnetic field limits, delta-shocks, vacuum states.

\section{REFERENCES}

[1] R. K. AgaRwal AND D. W. HaLt, A modified CUSP scheme in wavelparticle split form for unstructured grid Euler flows, Frontiers of Computational Fluid Dynamics, edited by D. A. Caughey and M. M. Hafes, John Wiley and Sons, 1994.

[2] F. Bouchut, On zero-pressure gas dynamics, Advances in kinetic theory and computing, Series on Advances in Mathematics for Applied Sciences, vol. 22, World Scientific, River Edge, NJ, 1994, pp. 171-190.

[3] Y. Brenier And E. Grenier, Sticky particles and scalar conservation laws, SIAM J. Numer. Anal. 35 (1998) 2317-2328.

[4] T. Chang And L. Hsiao, The Riemann Problem and Interaction of Waves in Gas Dynamics, Longman Scientific \& Technical, 1989.

[5] G. CHEN AND H. LIU, Formation of delta-shocks and vacuum states in the vanishing pressure limit of solutions to the isentropic Euler equations, SIAM J. Math. Anal. 34 (2003) 925-938.

[6] G. Chen AND H. LIU, Concentration and cavitation in the vanishing pressure limit of solutions to the Euler equations for nonisentropic fluids, Phys. D 189 (2004) 141-165.

[7] H. Cheng And H. YAng, Delta shock waves in chromatography equations, J. Math. Anal. Appl. 380 (2011) 475-485.

[8] H. Cheng AND H. YANG, Riemann problem for the relativistic Chaplygin Euler equations, J. Math. Anal. Appl. 381 (2011) 17-26.

[9] V. Danilov and V. Shelkovich, Dynamics of propagation and interaction of $\delta$-shock waves in conservation laws systems, J. Differential Equations 221 (2005) 333-381.

[10] R. J. DIPERNA AND A. MAJDA, Reduced hausdorff dimension and concentration-cancellation for two dimensional incompressible flow, J. Am. Math. Soc. 1 (1988) 59-59.

[11] P. LE FLOCH, An existence and uniqueness result for two nonstrictly hyperbolic systems, in Nonlinear Evolution Equations that Change Type, IMA 27 in Mathematics and its Applications, Springer-Verlag, 1990.

[12] C. Greengard And E. Thomann, On diperna-majda concentration sets for two-dimensional incompressible flow, Commun. Pur. App. Math. 41 (1988) 295-303.

[13] Y. B. Hu AND W. ShENG, The Riemann problem of conservation laws in magnetogasdynamics, Commun. Pure Appl. Anal. 12 (2013) 755-769. 
[14] K. T. Joseph, A Riemann problem whose viscosity solutions contain delta-measures, Asymptotic Anal. 7 (1993), 105-120

[15] B. L. KeYFITZ AND H. C. KRANZER, A viscosity approximation to system of conservation laws with no classical Riemann solution in Nonlinear Hyperbolic Problems, Lecture Notes in Mathematics, vol. 1042, Springer-Verlag, Berlin/New York, 1989.

[16] D. J. KORCHINSKI, Solutions of a Riemann problem for a $2 \times 2$ system of conservation laws possessing classical solutions, Adelphi University Thesis, 1977.

[17] S. KuIla AND T. RAJA SEKHAR, Riemann solution for ideal isentropic magnetogasdynamics, Meccanica 49 (2014) 2453-2465.

[18] S. KUILA AND T. RAJA SEKHAR, Riemann solution for one dimensional non-ideal isentropic magnetogasdynamics, Comp. Appl. Math. 35 (2016) 119-133.

[19] S. KUILA AND T. RAJA SEKHAR, Wave interactions in non-ideal isentropic magnetogasdynamics, Int. J. Appl. Comput. Math. 2016: 1-23.

[20] J. LI, Note on the compressible Euler equations with zero temperature, Appl. Math. Lett. 14 (2001) 519-523.

[21] Y. LI AND Y. CAO, Large particle difference method with second order accuracy in gas dynamics, Scientific Sinica (A) 28 (1985) 1024-1035.

[22] J. Li AND H. YANG, Delta-shocks as limits of vanishing viscosity for multidimensional zeropressure gas dynamics, Quart. Appl. Math. 59 (2001) 315-342.

[23] J. Li AND T. ZHANG, Generalized Rankine-Hugoniot relations of delta-shocks in solutions of transportation equations, Advances in Nonlinear Partial Differential Equations and Related Areas, World Sci. Publishing, River Edge, NJ, 1998.

[24] Y. LiU AND W. Sun, Riemann problem and wave interactions in Magnetogasdynamics, J. Math. Anal. Appl. 397 (2013) 454-466.

[25] A. Majda And G. Majda And Y. Zheng, Concentrations in the one-dimensional Vlasov-Poisson equations I: Temporal development and non-unique weak solutions in the single component case, Physica D 74 (1994) 268-300.

[26] T. Raja Sekhar And V. D. Sharma, Solution to the Riemann problem in a one-dimensional magnetogasdynamic flow, Int. J. Comput. Math. 89 (2012) 200-206.

[27] T. RAJA SEKHAR AND V. D. Sharma, Riemann problem and elementary wave interactions in isentropic magnetogasdynamics, Nonlinear Anal.-Real World Appl. 11 (2010) 619-636.

[28] S. F. SHANDARIN AND YA. B. ZELDOVICH, The large-scale structure of the universe: Turbulence, intermittency, structures in a self-gravitating medium, Rev. Mod. Phys. 61 (1989) 185-220.

[29] V. Shelkovich, The Riemann problem admitting $\delta, \delta^{\prime}$-shocks, and vacuum states (the vanishing viscosity approach), J. Differ. Equations 231 (2006) 459-500.

[30] W. ShENG AND T. ZHANG, The Riemann problem for the transportation equations in gas dynamics, Mem. Amer. Math. Soc. 137 (1999).

[31] D. TAN AND T. ZHANG, Two-dimensional Riemann problem for a hyperbolic system of nonlinear conservation laws I. Four-J cases, II. Initial data involving some rarefaction waves, J. Differ. Equations 111 (1994) 203-282.

[32] D. TAN AND T. ZHANG AND Y. ZHENG, Delta shock waves as limits of vanishing viscosity for hyperbolic systems of conservation laws, J. Differ. Equations 112 (1994) 1-32.

[33] E. WeinAn AND Y. G. RyKov AND Y. G. SinAi, Generalized variational principles, global weak solutions and behavior with random initial data for systems of conservation laws arising in adhesion particle dynamics, Coram. Math. Phys. 177 (1996) 349-380.

[34] H. YANG, Riemann problems for a class of coupled hyperbolic systems of conservation laws, J. Differ. Equations 159 (1999) 447-484.

[35] H. YANG AND J. LiU, Delta-shocks and vacuums in pressureless gas dynamics by the flux approximation, Sci. China Math. 58 (2015) 2329-2346.

[36] H. YANG AND J. LIU, Concentration and cavitation in the Euler equations for nonisentropic fluids with the flux approximation, Nonlinear Anal.-Theor. 123-124 (2015) 158-177.

[37] G. YIN AND W. Sheng, Delta shocks and vacuum states in vanishing pressure limits of solutions to the relativistic Euler equations for polytropic gases, J. Math. Anal. Appl. 355 (2009) 594-605.

[38] Y. Zheng, Systems of Conservation Laws: Two-Dimensional Riemann Problems, Birkhäuser, 2001. 